\title{
A rare and interesting case of desmoid-type fibromatosis arising from the fat layer of the abdominal wall: A case report
}

\section{Chuang Jiang}

Sichuan University West China Hospital

\section{Dan Xie}

Affiliated Hospital of North Sichuan Medical College

Hu Liu

Sichuan University West China Hospital

\section{Yunyou Tang}

West China School of Medicine: Sichuan University West China Hospital

\section{Mingheng Liao}

Sichuan University West China Hospital

Huaisheng Wang ( $\nabla$ wanghsmd@163.com)

Sichuan University West China Hospital

\section{Case report}

Keywords: Desmoid-type, Fibromatosis, Abdominal wall, Metastasis, Case report

Posted Date: May 6th, 2021

DOl: https://doi.org/10.21203/rs.3.rs-430714/v1

License: (c) (1) This work is licensed under a Creative Commons Attribution 4.0 International License. Read Full License 


\section{Abstract \\ Background}

Desmoid-type fibromatosis (DTF) is a rare kind of soft-tissue tumors of unknown origin, which is a benign mesenchymal neoplasm with monoclonal proliferation with local invasion, easy recurrence and nondistant metastasis. It is usually arising from the aponeurosis, muscle, and deep fascia, but DTF arsing from the fat layer have not been previously reported.

\section{Case presentation:}

A 22-year-old male was presented with a recurrence of lower abdominal wall mass(LAWM) after multiple operations and a new mass in the skin flap donor area of the right abdominal wall. Magnetic resonance imaging (MRI) revealed multiple soft tissue masses and nodules in the subcutaneous fat layer of the lower abdomen and right abdomen wall, which was considered a diagnosis of fibrosarcoma, then pathological consultation was performed on the paraffin specimens from previous operations, and finally confirmed the diagnosis of desmoid-type fibromatosis in combination with pathological characteristics of the resected specimens in this visit. In general, DTF did not have the ability to metastasize, but we extracted paraffin sections and performed full exon sequencing. After personalized analysis, the results showed that there was a possibility of implantative metastasis. Due to the aggressive growth and recurrence of the tumor, the patient was subsequently treated with radiotherapy and no recurrence was observed 6 months later. Continued follow-up is ongoing.

\section{Conclusions}

We report the first case of DTF that occurs in the superficial layer of fat with the possibility of surgically induced implantative metastasis, for such patients, diagnosis of DTF is more difficult and surgical treatment should be treated with greater caution.

\section{Background}

Desmoid-type fibromatosis (DTF) is a rare disease first reported in 1832[1], and was first named by Muller in 1938. This is a borderline soft tissue tumor usually arising from the aponeurosis, muscle, and deep fascia,also known as hard fibromatosis, invasive fibromatosis, aponeurosis fibromatosis or ligamentoid tumor, which is characterized by aggressive growth, postoperative recurrence, and non-metastasis[2]. The ratio of male to female in desmoid fibromatosis is about 1:3, mainly in women of childbearing age,the incidence is about $2-4 /$ million per year[3], which only accounts for $0.03 \%$ of solid tumors and $3 \%$ of soft tissue tumors[4]. According to the anatomic location of the disease, DTF were divided into 3 types: extraabdominal type, abdominal wall type, and intra-abdominal type[5].Mutations in CTNNB1 ( $\beta$-catenin gene), an important part of the Wnt pathway, lead to the accumulation of $\beta$-catenin in cells, which is closely 
related to the occurrence and prognosis of DTF[6].The etiology of desmoid fibroma is still not clearly, and it can occur in any part of the body[7-12]. For the treatment of desmoid fibromatosis, radical resection was used as the first-line treatment for a long time in the past. However, the local recurrence rate can be as high as $20 \%-65 \%$ within 5 years after resection[13], therefore, continuous follow-up and appropriate intervention are require.

\section{Case Presentation}

A 22-year-old man visited The West China Hospital of Sichuan University presented with a 3-year history of two progressive growing masses on the abdominal wall,one on the lower abdominal wall and the other on the right abdominal wall.These two masses protrude from the surface of the body without pain, itching, ulcers, or other symptoms.

When he was 17, he had his first operation at a local hospital to excise a soybean-sized mass on the lower abdominal wall in the fat layer, which was pathologically diagnosed as a "fibroma".

But a mass was found again at the healing site of the original surgical incision only 8 months later, protruding body surface, and gradually grew to the size of $6.5 \times 3.2 \mathrm{~cm}$. He visited another hospital asked for surgical treatment, the scope of surgical resection is still did not reach the anterior rectus sheath, however, due to the mass size is larger, the incision was wide and direct suture was difficult, so a medium thickness flap was taken from the right abdominal wall and transplanted to cover the incision at the lower abdomen. And he did not receive adjuvant therapy after operation. Postoperative pathologic report indicated spindle cell proliferative, in addition, there was no tumor invasion in the perioperative margins and basement of the surgical specimen.Further immunohistochemistry was performed and the results showed that:SMA (+), Desmin (-),S-100 (-), CD34 (-),ß-catenin (-), TLE-1 (-), EMA (-),Ki-67 (the positive rate was about $2 \%)$.

Due to the lack of clear diagnosis of DTF in the previous two visits to the hospital, the patient did not pay enough attention to the disease, so he did not receive regular follow-up.

Unfortunately, 3 years later, a recurrent mass was found in the lower abdominal incision, and it was larger than the one excised the second time, strangely, at the same time, a new,large and hard mass appeared in the incision of the right abdominal wall. Then, he came to plastic surgery department of our hospital(Fig.1) and underwent an enhanced magnetic resonance imaging (MRI) examination of abdomen(Fig.2A-F). It showed multiple soft tissue masses and nodules in the subcutaneous fat layer of the lower abdomen and right abdomen. T1WI(T1-weighted imaging) showed homogeneous medium signal, T2WI(T2-weighted imaging) showed low signal, uneven reinforcement after enhancement, the mass of lower abdominal wall grew invasively to the deep, part of which was not clearly demarcated from the anterior sheath of rectus abdominis, and the adjacent muscle fibers and fascia were thickened and enhanced. The radiologist considered the diagnosis of abdominal fibrosarcoma. 
In view of the slow growth, no obvious boundary, tough texture, and no obvious necrotic foci observed on $\mathrm{MRI}$, the masses is not fully consistent with the clinical characteristics of fibrosarcoma. At the same time, in order to confirm the pathological results of the two previously resected masses of the patient, we required the patient to go to the hospitals where he visited at that time to extract the paraffin section specimen for pathological consultation in the Pathology Department of West China Hospital. After consultation, the pathological results of the first two excised mass indicated that the spindle cell proliferative lesion supported the histological morphology of desmoid fibromatosis(Fig.3A,B).

For a definitive diagnosis, then we took some tissue from each of these masses and did a pathological examination(Fig 3C,D). The pathological report indicated that the two lesions were consistent, both were spindle cell proliferative lesions and fibrogenic tumors, scar tissue can be seen in some superficial areas (consistent with keloid). Further immunohistochemical analysis results were as follows: the pathological cells showed desmin (-), SMA (+), $\beta$-cantenin (nuclear -), myo D1 (-), CD34 (-), EMA (-), TLE-1 (-), MUC-4 (-). CTNNB1 gene mutation: Exon3 mutation was not detected. The histological morphology of the two masses previous resected was reviewed, which was consistent with the specimen sent for biopsy, the final diagnosis was desmoid fibromatosis through comprehensive analysis.

After the definitive diagnosis, we detailed the risks associated with surgery and recurrence of the masses to the patient and signed an informed consent form for the operation. Considering that the two tumors were too large, the enlarged resection might be directly difficult to suture, and if the skin flap was transplanted to cover the wound, a new tumor might appear in the donor area again, so we chose to excise the tumor along the edge of the tumor. Intraoperatively, it was found that the mass of the lower abdominal wall was mostly located in the adipose layer, and a small part of the mass in the deep side invaded the anterior sheath of rectus abdominis. Therefore, we excised the invaded anterior sheath of rectus abdominis, and no invasion of muscle tissue was observed(Fig.4A-C). The right abdominal wall mass(RAWM) only extends to the adipose layer. We excised the mass completely but did not excise a portion of scar tissue on the right abdominal wall that did not touch the obvious mass(Fig.4D-F), then sutured both incisions directly. The patient was discharged from hospital after incision healing(Fig.5).

The pathological result showed that both tumors were subcutaneous, grayed white cut surface, solid and tough texture. The diagnosis of desmoid fibromatosis was still considered in combination with the biopsy result(Fig.6A,B).

The surgical incisions healed and sutures were dismantled 15 days after surgery, then he went to the oncology department and received radiation therapy. Image guided IMRT(Intensity-modulated radiation therapy) method was adopted. The radiotherapy regimen was described below:D95\% CTV(clinical target volume) 1,2(Postoperative tumor bed area) 1.5Gy/fraction,bid(bis in die). The radiotherapy went smoothly 30 times. The patient developed an ulcer on the skin of the lower abdominal wall after radiotherapy,which healed after active dressing change.

In general, DTF did not have the ability to metastasize,but the emergence of the mass in the right abdominal wall caught our attention. We extracted paraffin sections of the two surgical specimens from 
the Department of Pathology. After evaluating the paraffin samples, it was concluded that whole-genome sequencing might not be as effective as expected, we finally chose the relatively effective whole exon sequencing.

The sequencing results as follows: the SNV(single nucleotide variant) mutaition and the InDel (insertion and deletion) mutation of the two masses were very similar(Fig. S1), and the consequence of the tumor variant types were similar(Fig. S2).

After mutation filtration, a total of 41 somatic SNV and InDel mutations were found in paraffin section samples of right abdominal wall mass(RAWM), and 20 in lower abdominal wall mass(LAWM), due to there were fewer high quality somatic cell mutations detected, we tested the homology of the two masses according to the method reported in previous study[14]. Somatic mutations in the two samples contained 13 non-synonymous mutations in RAWM and 6 non-synonymous mutations in LAWM. And there were 4 shared mutations between the two samples(Fig.7). Shared mutations occupy $30.8 \%$ (RAWM) and $66.7 \%$ (LAWM) of the two samples, respectively. According to the reporting criteria, there was more than one shared mutation, so it can be judged as a implantative metastasis.

Six months after the radiotherapy, abdominal MRI reexamination showed a small amount of enhanced signals in the operative area, scar tissue was considered, and no obvious tumor recurrence was observed. During regular follow-up of patients,the patient's abdominal wall was in good condition and no neoplastic mass was observed in the operation area and follow-up is continuing.

\section{Discussion And Conclusions}

DTF is a rare interstitial neoplasm which usually originated from muscle, aponeurosis, and deep fascia[15]. The development of abdominal wall type DTF in most patients is associated with trauma to the musculofascial structure[16], but the previous two resection of this case only reached the depth of the fat layer and did not injure the rectus abdominis or anterior sheath. More significant, this is the first reported case of DTF occurring in the fat layer.

Although the area where the skin flap was taken only damaged the subcutaneous fat and did not reach the deeper muscle or fascia, this patient developed a new mass similar to the lower abdominal wall on the right abdominal wall, which aroused our keen interest in the cause of the new tumor. Therefore, in order to explore the origin relationship between the two tumors, we performed exon sequencing on the obtained paraffin sections (10um/ sheet $\times 10$ sheets). The result shows the two masses met the conditions of local metastasis according to the reported criteria[14]. We ventured to speculate that since DTF is generally considered to be non-metastatic, the surgical blade may not be replaced when the skin flap was obtained in the last operation, which may lead to the implantation of tumor cells. However, it is a pity that we did not retain the fresh frozen tissue in time, and the accuracy of the results obtained by the total exon sequencing of the paraffin section specimens was lower than that of the frozen specimens. Besides, as we dare not expand or add new surgical incisions at will, normal skin tissue samples of this patient were not obtained, so CNV(copy number variation) was not used to analyze and verify whether 
implantative metastasis was present. But in view of benign tumors, there is usually less somatic CNV, therefore, this result has reference significance but still needs to be further verified.

Pathology is the gold criteria for the diagnosis of DTF. Mutations in CTNNB1( $\beta$-catenin gene) lead to the accumulation of $\beta$-catenin in cells,and immunohistochemistry showed positive for $\beta$-catenin. Approximately $33 \%$ to $100 \%$ of DTFs test positive for $\beta$-cantenin $[6,17,18]$. However, in this case, no mutation was detected in CTNNB1, $\beta$-cantenin was negative and only SMA was positive by immunohistochemistry. This makes the diagnosis of the pathologist a little more difficult due to the atypical immunohistochemical results. For these patients, careful differentiation with other tumors is needed to avoid misdiagnosis

A study[19] of 495 patients showed that risk factors for postoperative recurrence included age (<26y), tumor size $(>10 \mathrm{~cm})$, resection margins in tumours $<5 \mathrm{~cm}$. Postoperative radiotherapy is necessary in patients with incomplete resection or a history of local recurrence[20, 21]. Although the tumors was completely excised in this case, due to his young age, the tumor $>10 \mathrm{~cm}$ and with a history of recurrence, we recommended that he receive radiotherapy, although there was a skin ulcer in the radiotherapy area, but recovered after active treatment. After 6 months of follow-up, no recurrence was found.

In conclusion, we have reported a unique case of DTF that is difficult to diagnose, and the highlight of this case is that we found and reported for the first time that DTF may also originate from superficial tissues rather than deeper muscles and fascia, requiring careful differentiation from cutaneous fibrous neoplasms. For patients with DTF whose tumors occurred in the subcutaneous fat layer may be more sensitive to superficial tissue damage, surgeons should probably try to avoid surgery and adopt a "waitand-see" strategy instead. But, when the tumor continues to grow and there is no stabilization trend, surgery become necessary. We should minimize surgical incision in order to avoid damage to normal tissues and lead to new tumor. If the tumor is large enough that the incision cannot be sutured directly after resection, a skin flap should be taken to cover the wound, and a new surgical blade must be replaced in order to prevent the implantation of tumor cells.

\section{Abbreviations}

RAWM:right abdominal wall mass, LAWM: lower abdominal wall mass, IMRT:Intensity-modulated radiation therapy, CTV: clinical target volume, bid: bis in die, SNV: single nucleotide variant, CNV: copy number variation, InDel: insertion and deletion, MRI: Magnetic resonance imaging, T1WI:T1-weighted imaging, T2Wl:T2-weighted imaging

\section{Declarations}

Acknowledgments

Not Applicable. 


\section{Authors' contributions}

Conception and design: CJ, DX, HL, YYT. Drafted the initial manuscript: CJ, DX. Analysis and interpretation of the data:MHL. Performed the surgery,postoperative management and follow-up: CJ,HSW. Analyses, reviewed and revised the manuscript:HSW. All authors read and approved the final manuscript.

\section{Funding}

This work is supported by the 1.3.5 project for disciplines of excellence-West China Hospital,Sichuan University.The organization was not involved in the treatment of patient, the design of the study, or the writing of the manuscript.

\section{Availability of data and materials}

The data used during the current study will be available upon request to the corresponding author on reasonable request.

\section{Ethics approval and consent to participate}

The Ethics Committee of Sichuan University approved the study.

\section{Consent for publication}

Written informed consent was obtained from the patient for the publication of the manuscript and accompanying images.

\section{Competing interests}

The authors declare that they have no competing interests.

\section{Author details}

${ }^{1}$ Department of Liver Surgery, Liver Transplantation Center, West China Hospital, Sichuan University, Chengdu Sichuan, 610041 ,China. ${ }^{2}$ Department of Burns and Plastic Surgery, West China Hospital, Sichuan University, Chengdu Sichuan, 610041 ,China. ${ }^{3}$ Department of Anesthesiology, Affiliated Hospital of North Sichuan Medical College, Nanchong Sichuan,637100, China. ${ }^{4}$ West China School of Preclinicalmedicine and Forensic Medicine, Sichuan University,Chengdu Sichuan, 610041,China

\section{References}

1. Macfarlane J. Clinical reports of the surgical practice of the Glasgow Royal Infirmary. D Robertson Glasgow. 1832;63-66.

2. Fletcher C. The evolving classification of soft tissue tumours - an update based on the new 2013 WHO classification. Histopathology. 2014;64(1):2-11.

3. Smith A, Lewis J, Merchant N, Leung D, Woodruff J, Brennan M. Surgical management of intraabdominal desmoid tumours. The British journal of surgery. 2000;87(5):608-13. 
4. Jo V, Fletcher C. WHO classification of soft tissue tumours: an update based on the 2013 (4th) edition. Pathology. 2014;46(2):95-104.

5. Kreuzberg B, Koudelova J, Ferda J, Treska V, Spidlen V, Mukensnabl P. Diagnostic problems of abdominal desmoid tumors in various locations. European journal of radiology. 2007;62(2):180-5.

6. Martínez Trufero J, Pajares Bernad I, Torres Ramón I, Hernando Cubero J, Pazo Cid R. Desmoid-Type Fibromatosis: Who, When, and How to Treat. Current treatment options in oncology. 2017;18(5):29.

7. Tsukamoto Y, Imakita M, Nishitani A, Ito T, Izukura M, Hirota S. Pancreatic desmoid-type fibromatosis with beta-catenin gene mutation-Report of a case and review of the literature. Pathology research practice. 2016;212(5):484-9.

8. Monaco F, Monaco M, Barone M. Aggressive and recurrent desmoid-type fibromatosis of the neck. Asian cardiovascular thoracic annals. 2015;23(8):1002.

9. Choi K, An Y. Desmoid Tumor of the Chest Wall Mimicking Recurrent Breast Cancer: Multimodality Imaging Findings. Iranian journal of radiology: a quarterly journal published by the Iranian Radiological Society. 2016;13(4):e31649.

10. Nakanishi K, Shida D, Tsukamoto S, et al. Multiple rapidly growing desmoid tumors that were difficult to distinguish from recurrence of rectal cancer. World journal of surgical oncology. 2017;15(1):180.

11. Yamamoto R, Mokuno Y, Matsubara H, Kaneko H, lyomasa S. Multiple mesenteric desmoid tumors after gastrectomy for gastric cancer: A case report and literature review. International journal of surgery case reports. 2018;50:50-5.

12. Navin P, Lehrke H, Schmitz J, Truty M, Moynagh M. Desmoid Fibromatosis Mimicking Metastatic Recurrence After Pancreatectomy for Pancreatic Adenocarcinoma. Mayo Clinic proceedings Innovations, quality \& outcomes. 2018;2(4):392-397.

13. Bonvalot S, Desai A, Coppola S, et al. The treatment of desmoid tumors: a stepwise clinical approach. Annals of oncology: official journal of the European Society for Medical Oncology. 2012:x158-166.

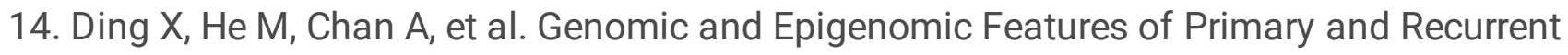
Hepatocellular Carcinomas. Gastroenterology. 2020;157(6):1630-45.

15. Jafri S, Obaisi O, Vergara G, et al. Desmoid type fibromatosis: A case report with an unusual etiology. World journal of gastrointestinal oncology. 2017;9(9):385-9.

16. Kasper B, Ströbel P, Hohenberger P. Desmoid tumors: clinical features and treatment options for advanced disease. The oncologist. 2011;16(5):682-93.

17. Stone A, Mallery J, Stewart J, Amin K. A rare sporadic pancreatic desmoid fibromatosis diagnosed by endoscopic ultrasound-guided fine-needle aspiration: Case report and literature review. Diagnostic cytopathology. 2021;49(2):E49-54.

18. Sabharwal S, Ahlawat S, Levin A, et al. Desmoid tumor mimics local recurrence of extremity sarcoma on MRI. Journal of surgical oncology. 2020;121(8):1259-65. 
19. Crago A, Denton B, Salas S, et al. A prognostic nomogram for prediction of recurrence in desmoid fibromatosis. Annals of surgery. 2013;258(2):347-53.

20. Nuyttens J, Rust $P$, Thomas $C$, Turrisi A. Surgery versus radiation therapy for patients with aggressive fibromatosis or desmoid tumors: A comparative review of 22 articles. Cancer. 2000;88(7):1517-23.

21. Keus R, Nout R, Blay J, et al. Results of a phase II pilot study of moderate dose radiotherapy for inoperable desmoid-type fibromatosis-an EORTC STBSG and ROG study (EORTC 62991 - 22998). Annals of oncology: official journal of the European Society for Medical Oncology. 2013;24(10):2672-6.

\section{Figures}

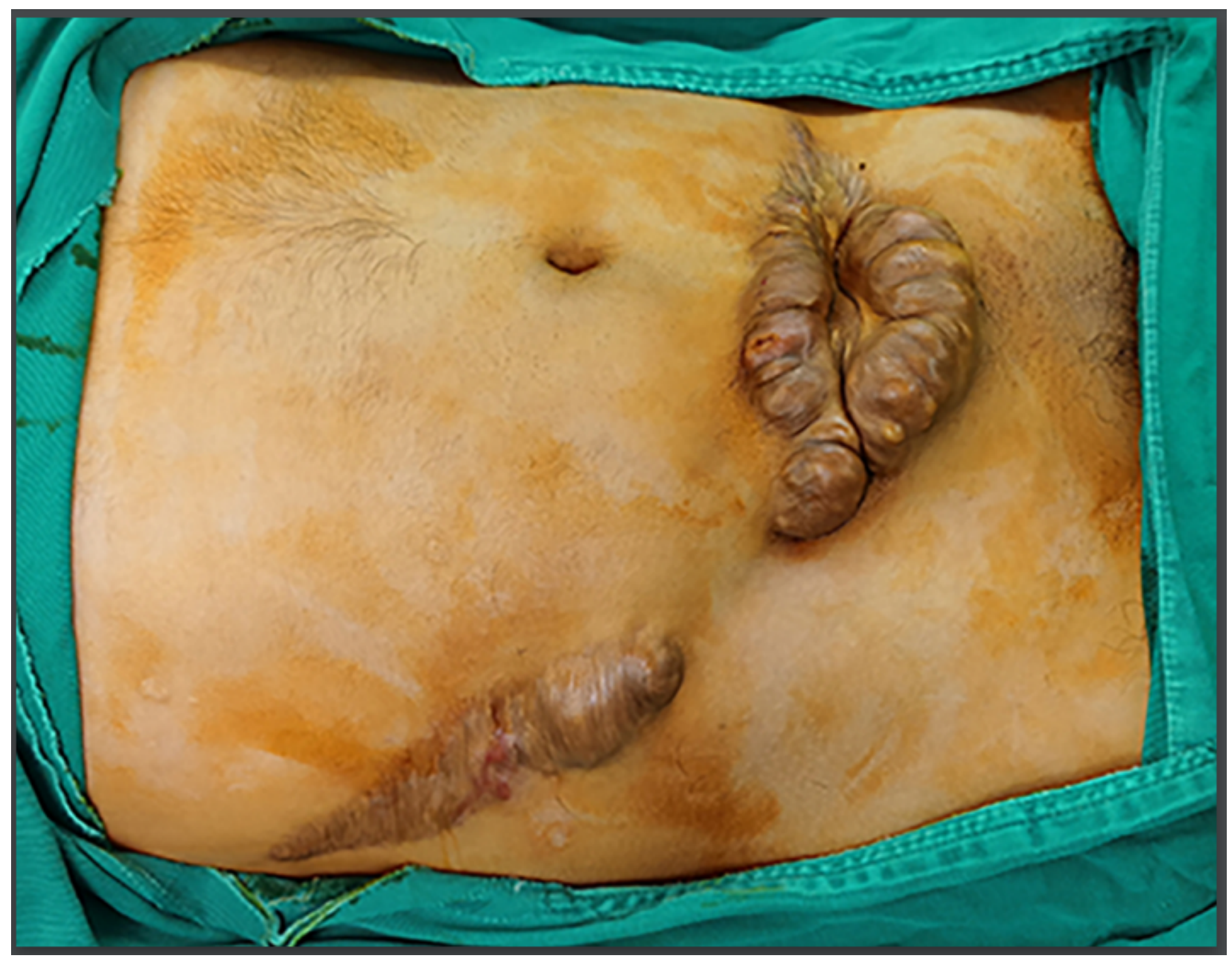

\section{Figure 1}

Clinical examination A $13.5 \times 8.5 \mathrm{~cm}$ hard, irregular shape and lobulated mass in lower abdominal wall; a $8.0 \mathrm{~cm} \times 3.0 \mathrm{~cm}$ hard mass in right abdominal wall. 


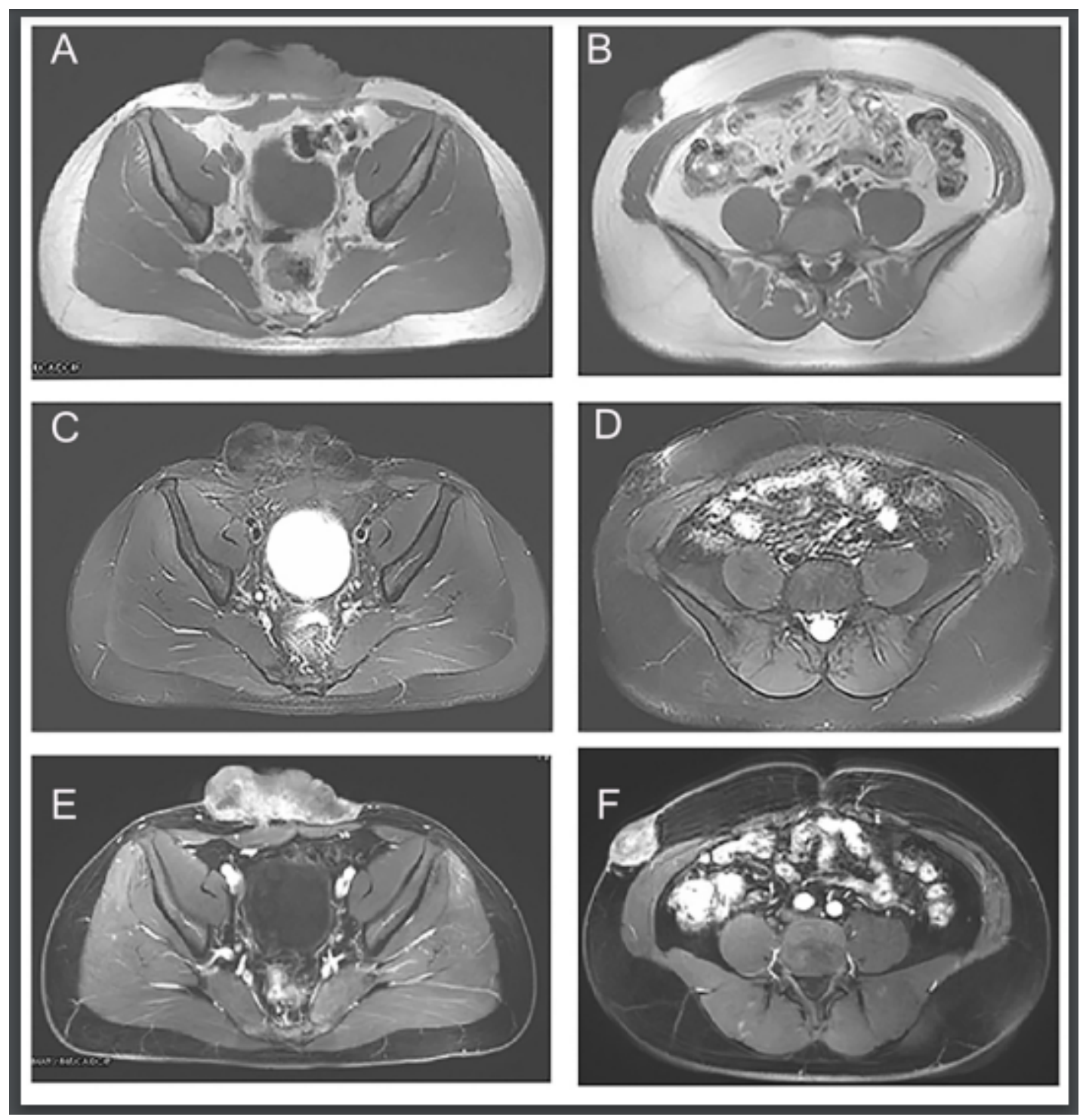

\section{Figure 2}

MRI of lower abdominal wall and right abdominal wall masses $(A)$ :The lower abdominal wall mass(LAWM) showed homogeneous medium signal intensity on $\mathrm{T} 1 \mathrm{Wl}$, a large portion of which located in the adipose layer and grow invasively to the deep, with an unclear local boundary with the anterior sheath of the rectus abdominis; (B) :The right abdominal wall mass(RAWM) showed homogeneous medium signal intensity on T1WI, completely within the fat layer; (C) :The LAWM showed hypointense on T2Wl; (D) :The RAWM showed hypointense on T2Wl; (E) :The LAWM showed mixed signal with uneven enhancement; $(F)$ :The RAWM showed mixed signal with uneven enhancement. 


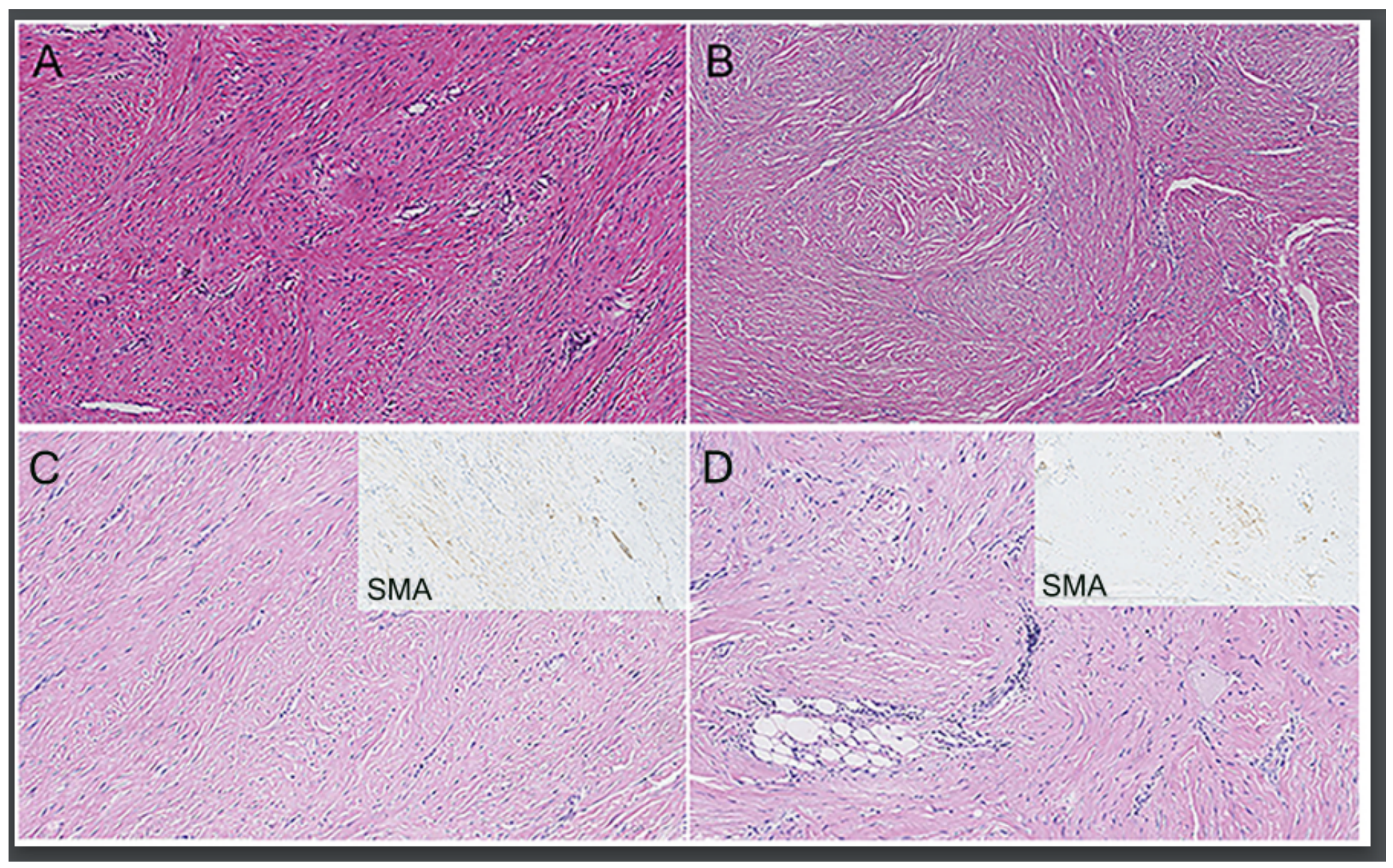

\section{Figure 3}

Histopathological findings (A and B): Haematoxylin-eosin staining (Paraffin section of the first two surgical specimen). Proliferative spindle cells in a sheet-striated or interlaced arrangement (No obvious

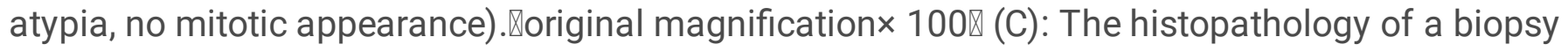
specimen of LAWM showed proliferative spindle cells arranged interlaced in collagenous background and positive for SMA囚original magnification $\times 100 \otimes(D)$ :The histopathology of a biopsy specimen of RAWM showed an abundance of spindle cells,displaying virtually no mitotic activity , abundant stromal collagen

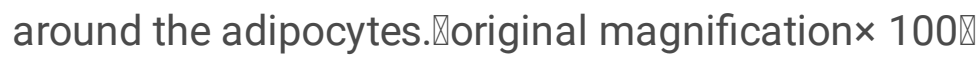




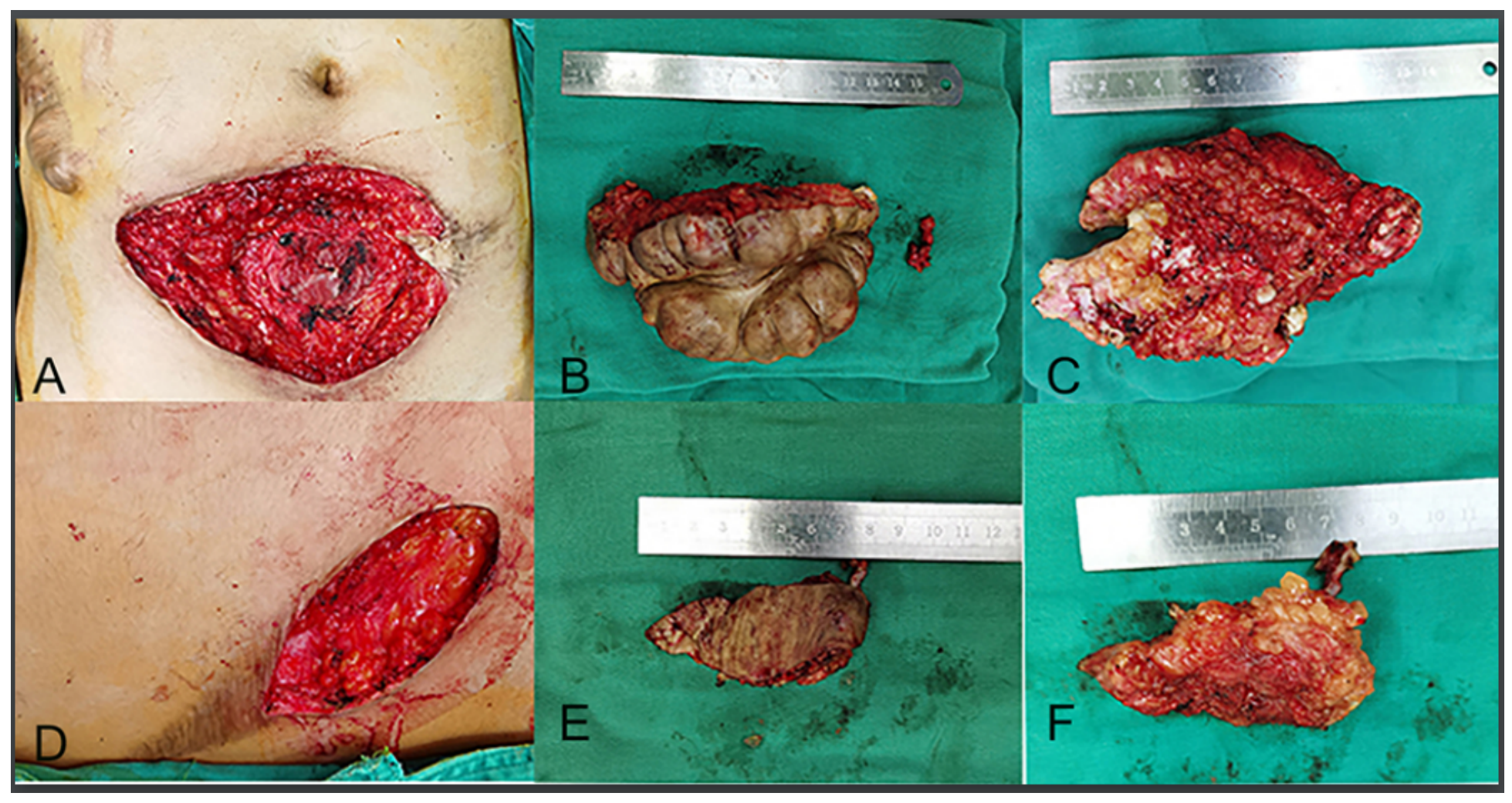

Figure 4

Intraoperative findings A,B and C: Intraoperatively, the mass of the lower abdominal wall was mainly located in the fat layer,then the mass and the affected anterior rectus sheath were completely resected. $\mathrm{D}, \mathrm{E}$ and $\mathrm{F}$ : The right abdominal wall mass was all located within the fat layer, the mass was completely excised, and portion of scar tissue that did not touch the obvious mass was left. 


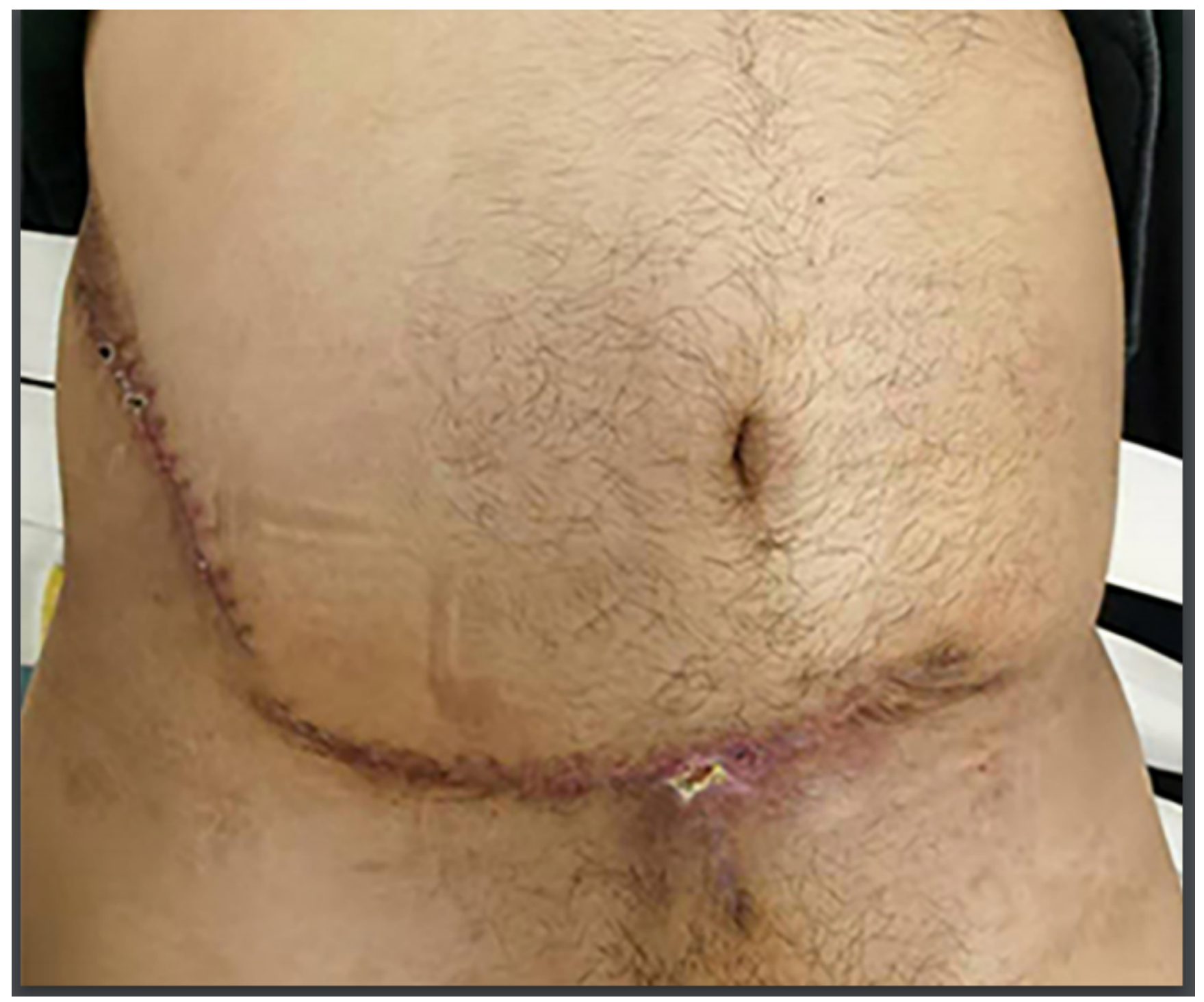

Figure 5

The incision healed well

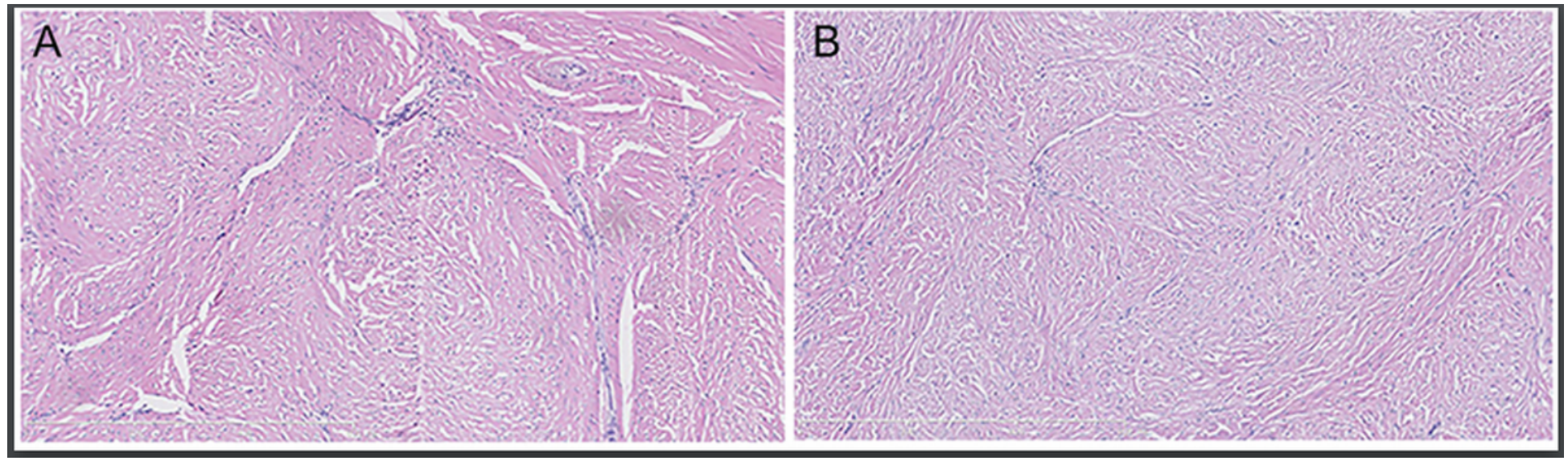

Figure 6 
Histopathology of resected mass Proliferative spindle cells arranged interlaced in a fibrotic background(hematoxylin and eosin, original magnification $\times 100$.(A) The histopathology of LAWM ;(B)The histopathology of RAWM.

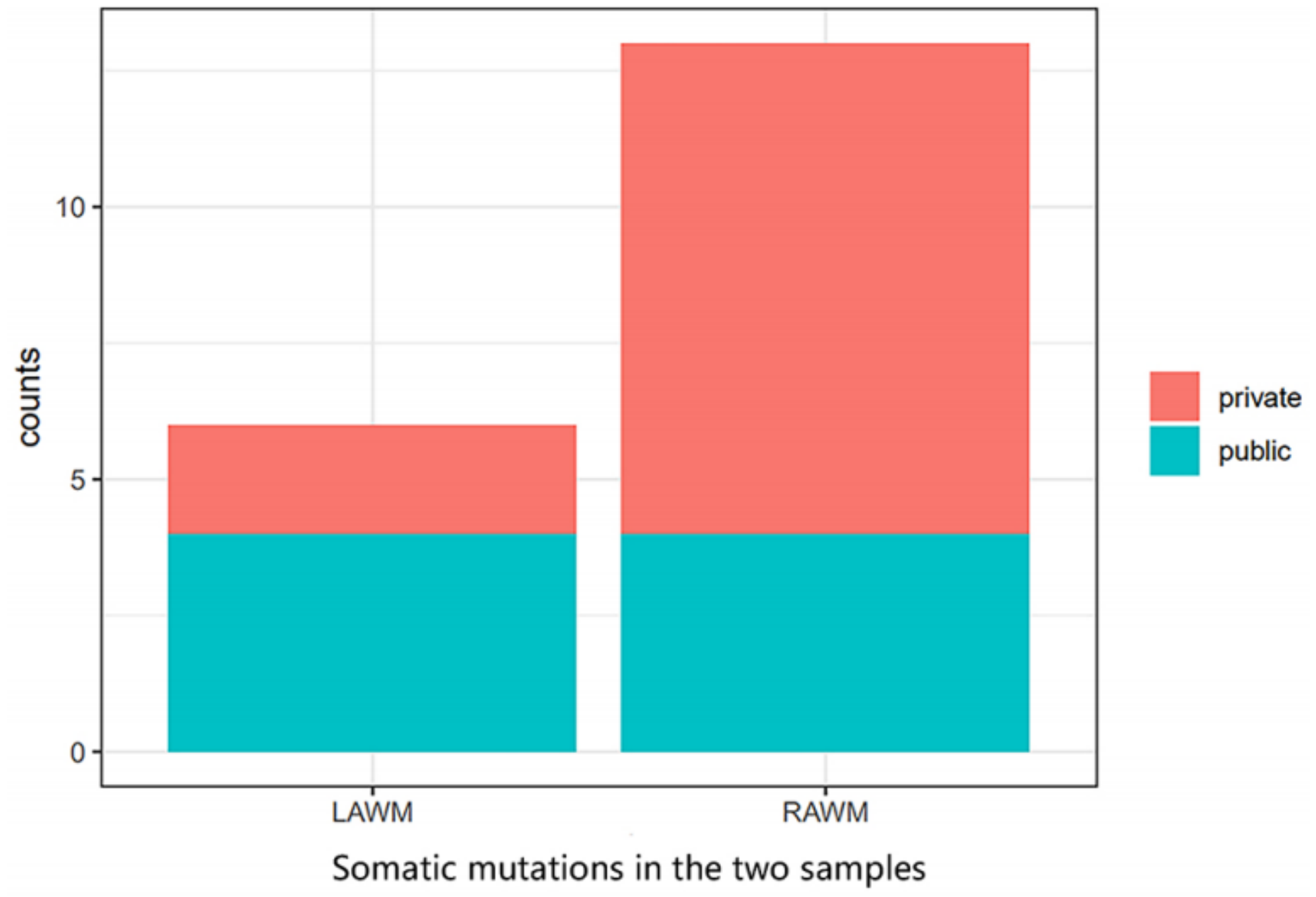

Figure 7

Mutations in these two samples Somatic mutations contained 13 non-synonymous mutations in RAWM and 6 non-synonymous mutations in LAWM. 4 shared mutations were found.

\section{Supplementary Files}

This is a list of supplementary files associated with this preprint. Click to download.

- SupplementaryFigure1.pdf

- SupplementaryFigure2.pdf

- CAREchecklistEnglish2013.pdf 